\title{
Risk assessment of ayahuasca use in a religious context: self-reported risk factors and adverse effects
}

\author{
ícaro Durante, ${ }^{1}$ (iD Rafael G. dos Santos, ${ }^{2,3,4}$ (iD José C. Bouso, ${ }^{4}$ (iD Jaime E. Hallak ${ }^{2,3}$ (iD \\ ${ }^{1}$ Faculdade de Medicina, Universidade Federal da Fronteira Sul (UFFS), Passo Fundo, RS, Brazil. ${ }^{2}$ Departamento de Neurociências e \\ Ciências do Comportamento, Faculdade de Medicina de Ribeirão Preto, Universidade de São Paulo (USP), Ribeirão Preto, SP, Brazil. \\ ${ }^{3}$ Instituto Nacional de Ciência e Tecnologia Translacional em Medicina, Ribeirão Preto, SP, Brazil. ${ }^{4}$ International Center for Ethnobotanical \\ Education, Research and Services (ICEERS), Barcelona, Spain.
}

\begin{abstract}
Objective: Whether for spiritual, recreational, or potential therapeutic use, interest in ayahuasca has grown remarkably. Ayahuasca's main active substances are $N, N$-dimethyltryptamine and certain monoamine oxidase inhibitor $\beta$-carbolines. Possible drug interactions are a major concern, and research is lacking in this area. The objective of this study was to evaluate the safety of ritual ayahuasca use regarding adverse effects and risk factors.

Methods: In this cross-sectional study, ayahuasca users from a religious institution answered an online questionnaire about its safety. Adverse effects, safety measures, and possible risk factors (psychiatric diagnosis and medications) were investigated.

Results: The most frequent adverse effects among the 614 participants were transient gastrointestinal effects (nausea and vomiting). Fifty participants self-reported a psychiatric diagnosis (depression and anxiety were the most prevalent), and these participants experienced adverse effects more frequently. Psychiatric medication use was reported by 31 participants. No indication of increased adverse effects due to drug-drug interactions was found.

Conclusion: A minority of participants reported being very negatively affected by persistent adverse effects. Psychiatric medication use while participating in ayahuasca rituals was not associated with increased adverse effects. For the most part, the institution's practices seem sufficient to prevent exacerbated reactions. Future studies may focus on negatively affected users.
\end{abstract}

Keywords: Ayahuasca; adverse effects; monoamine oxidase inhibitor; risk factors; harm reduction

\section{Introduction}

Ayahuasca is a psychoactive brew traditionally used by indigenous Amazonian cultures for ritual and medical purposes. More recently, it became a ceremonial sacrament for Brazilian syncretistic religions, such as Santo Daime, Barquinha, and União do Vegetal (UDV). ${ }^{1,2}$ This study was conducted with members of UDV, in which ayahuasca is also called Hoasca and Vegetal, and is consumed ritualistically to promote a non-ordinary state of consciousness that can be described as "mental concentration," around which the religious practices revolve. ${ }^{2}$ UDV members can attend regular bimonthly sessions at the existing centers, as well as extra sessions on special occasions. The distribution of ayahuasca during the sessions is supervised by an experienced member, the Mestre, who guides the religious ceremony. ${ }^{2}$ Currently, UDV is present in 11 countries, with more than 21,000 associated members. ${ }^{3}$ The brew used by UDV members is prepared

Correspondence: Ícaro Durante, Universidade Federal da Fronteira Sul, Faculdade de Medicina, Rua Capitão Araújo, 20, CEP 99010200, Passo Fundo, RS, Brazil.

E-mail: icaro@estudante.uffs.edu.br

Submitted Feb 16 2020, accepted Jul 16 2020, Epub Oct 302020. with a typical combination of Banisteriopsis caapi and Psychotria viridis, also known as Mariri and Chacrona, respectively. ${ }^{4}$ By the decoction of these Amazonian plants, their active substances are extracted. ${ }^{1,4}$ The main component of ayahuasca is the alkaloid $\mathrm{N}, \mathrm{N}$-dimethyltryptamine (DMT), a tryptamine present in the leaves of Psychotria viridis. $^{5}$ This tryptamine acts predominantly as a $5-\mathrm{HT}_{2 \mathrm{~A}}$ receptor agonist, ${ }^{6}$ with effects comparable to those of lysergic acid diethylamide, psilocybin, or mescaline. ${ }^{7}$

DMT is not orally active on its own, since it is metabolized by peripheral monoamine oxidase enzymes (MAO), especially the MAO-A subtype. ${ }^{6}$ These enzymes occur across several tissue types, and by catalyzing the oxidation of amines ${ }^{8}$ they have an important role in degrading neurotransmitters. Ayahuasca also includes alkaloids that act as MAO inhibitors (MAOI), namely harmaline, harmine, and tetrahydroharmine. These substances, extracted from Banisteriopsis caapi, belong to a group of $\beta$-carbolines known as harmala alkaloids. ${ }^{1,9}$ The presence of these

How to cite this article: Durante I, dos Santos RG, Bouso JC, Hallak JE. Risk assessment of ayahuasca use in a religious context: self-reported risk factors and adverse effects. Braz J Psychiatry. 2021;43:362-369. http://dx.doi.org/10.1590/1516-4446-2020-0913 
MAOIs in ayahuasca prevents the degradation of DMT, enabling its absorption into systemic circulation and the central nervous system. ${ }^{1,9}$

Several conventional drugs exhibit some degree of MAO inhibition, and this effect was exploited by the first antidepressants. ${ }^{10}$ Inhibition of this enzyme results in an accumulation of the substances it usually degrades, including endogenous neurotransmitters. ${ }^{11}$ If the inhibition is too intense or sustained, the increased concentration of monoamines can cause systemic manifestations. When combined with monoaminergic substances, some MAOIs can cause deleterious reactions. ${ }^{12,13}$ One particularly worrisome outcome of inhibiting MAO enzymes, especially in combination with serotonergic agents, is the accumulation of serotonin to toxic levels, a condition known as "serotonin syndrome," which, although rare, can be fatal. ${ }^{13}$

The influence of the MAO inhibition through ayahuasca's $\beta$-carbolines is still poorly understood. Concern about the interaction of these MAOls with other substances is due to research on conventional MAOIs, especially older antidepressants. ${ }^{14}$ Thus, the risk factors and contraindications generally proposed for ayahuasca are based on the effects and interactions observed from the combination of these medications with monoaminergic drugs, such as selective serotonin reuptake inhibitors, and foods rich in amines, such as aged cheeses and red wine, due to their tyramine content. ${ }^{11,14,15}$ The $\beta$-carbolines in ayahuasca act as selective (mainly on MAO-A) and reversible MAOIs, which could render these alkaloids safer than unspecific and irreversible MAOIs. ${ }^{9,15}$ Regardless of the mechanisms of action of each harmala alkaloid, their MAO-inhibiting properties and the indispensability of this condition for the psychotropic effects of ayahuasca are well-grounded. ${ }^{9}$

The subjective effects of ayahuasca are dose-dependent and closely related to serum DMT concentrations. ${ }^{16}$ These effects usually become noticeable 30-60 minutes after administration, peaking between 60-120 minutes, with an overall duration of 240 minutes. ${ }^{16}$ Effects on perception, such as characteristic visual imagery ("visions"), are consistently reported by most users. The perceptual alterations are accompanied by changes in cognition, affect, and volition. ${ }^{16}$ Many of these effects can attain spiritual importance in a religious context.

Common acute physical effects include nausea, vomiting, and diarrhea, which could be a result of increased levels of serotonin in the gastrointestinal tract. ${ }^{17}$ Cardiac effects such as increased blood pressure and heart rate may also occur and could be attributed to decreased degradation (and, thus, accumulation) of catecholamines, such as norepinephrine. ${ }^{17}$

Studies on ayahuasca and its safety were performed during the 1990s with UDV members in what came to be known as Hoasca Project. This project provided ayahuasca research some of its seminal works, with preliminary observations about short- and long-term effects. These investigations found no evidence of physical/ psychological dependence or permanent sequelae due to long-term ayahuasca use in a religious context. ${ }^{1,17}$

In 1998, Callaway \& $\mathrm{Grob}^{14}$ described the case of a benign serotonin syndrome, possibly induced by the interaction between ayahuasca and a selective serotonin reuptake inhibitor. This is the only case of such a reaction identified in the literature. Indeed, concomitant use of ayahuasca with other drugs and medications is not advised. Studies to establish the safety of ayahuasca in such situations are still required, especially due to the increasing number of users. These studies should investigate whether the previously recognized risk factors of conventional MAOls apply to ayahuasca and to what degree, as well as whether the institution's safety measures can prevent serious adverse reactions.

The main objective of this study was to assess the risk profile of ayahuasca use in a religious context. After reviewing the literature to identify important concerns and common adverse effects that could be self-reported, an instrument was developed that inquired about the frequency of the following effects: anxiety, diaphoresis, diarrhea, disorientation, distress, dizziness, drowsiness, dry mouth, headache, irritability, nausea, restlessness, shivers, tachycardia, tinnitus, tremor, and vomiting. Psychiatric diagnoses and psychiatric medication use were also considered risk factors due to their possible interactions with the MAOls in ayahuasca. The existence and implementation of safety measures were also investigated. The collected data were analyzed to test the hypothesis that users with risk factors experience adverse effects more frequently, which could indicate that drug interactions elevate the concentration of monoamines to toxic levels, or have a negative influence on subjacent psychiatric conditions.

\section{Methods}

This cross-sectional study was conducted with members of UDV centers in the states of Paraná, Rio Grande do Sul, and Santa Catarina. According to the organization's internal records, these centers have a total of 1,547 associated members (UDV internal data; information granted March 2019), in addition to an undetermined number of non-associated attendees. The only exclusion criteria were age less than 18 years and never having used ayahuasca.

A questionnaire was developed on the use of MAOls and psychedelics/hallucinogens in a religious context based on needs identified in the literature. ${ }^{8,14-16}$ The questionnaire was designed to gather basic sociodemographic data and describe relevant aspects of ayahuasca use, especially the general safety of MAOls, characteristics suggestive of an increased risk of severe reactions, and adverse effects common to serotonin toxicity. Therefore, questions were designed to explore the frequency of ayahuasca use, medication use (mainly psychiatric), history of psychiatric disorders, and perceived adverse effects and their persistence, in addition to satisfaction regarding UDV safety measures. ${ }^{14-17,18,19}$

The development of the questionnaire carefully considered how incisively the questions could be interpreted and how they might precipitate judgment. Most opinion questions were designed as five-point Likert scales, and the participants were prompted to report a psychiatric diagnosis only if it had been confirmed by a licensed 
professional. The digital, self-applied questionnaire was shared and answered through an online link, ensuring a broad reach and easy availability. The digital form employed conditional logic to guide respondents through sections of questions, covering a broad range of topics while avoiding an excessive burden. Since the collected data may not thoroughly address some important topics, further investigation is required.

The questionnaire was distributed with the collaboration of volunteers from the UDV medical and scientific department, which represents UDV in the scientific community. Each center had a designated volunteer responsible for promoting the survey and sharing its link through regular UDV communication channels. Thus, the questionnaire was distributed mainly through social network groups. Through the online link, interested members were led to an informed consent form explaining the nature of the study and, after completing it, they proceeded to the questionnaire.

The participants were not necessarily invited directly, but were exposed to information about the survey in group meetings and online spaces commonly frequented by UDV members. Considering that ayahuasca users are a hard-to-reach population, this self-selection method was the only viable approach. Alternative sampling would have required disclosure of known UDV members, violating their right to privacy. The questionnaire remained open and accepted responses for a period of 7 weeks between February and March 2019, with periodic reinforcement of outreach efforts.

The collected responses were imported into LibreOffice Calc for data homogenization and preparation. GNU PSPP was then used to calculate the relative and absolute frequency distributions of the variables. Pearson's chi-square test of independence and Fisher's exact test were used to analyze variables of interest and identify differences between groups.

Because the $\beta$-carbolines present in ayahuasca may affect the degradation of monoamines, the concomitant use of serotonergic agents could result in symptoms of serotonin toxicity. Our hypothesis was that concomitant use of antidepressant medication, due to its monoaminergic action, could increase the frequency of reported adverse effects. This would suggest a possible interaction between antidepressants and the MAOls present in ayahuasca. In addition, the frequency of adverse effects reported by participants with and without a psychiatric diagnosis was also compared. Finally, risk reduction measures were evaluated retrospectively. P-values < 0.05 were considered statistically significant.

\section{Ethics statement}

All participants provided electronic informed consent prior to accessing the questionnaire. This study was approved by the Universidade Federal da Fronteira Sul research ethics committee (CAAE: 02039218.5.0000.5564).

\section{Results}

The sample consisted of 614 participants who agreed to answer the questionnaire after providing electronic informed consent. A total of 606 participants $(99.7 \%$ of the sample) reported being members of one of the UDV centers in the three states investigated in the study, thus, the sample corresponds to $39.17 \%$ of the total population of UDV's associate members in these states at that time. The remaining members may have declined to participate or were not reached during efforts to promote the survey. All 15 centers in the three-state area were represented, as well as one authorized distribution center.

The basic sociodemographic profile of the participants was similar to previous findings for Brazilian UDV members, suggesting nationwide homogeneity in this population. ${ }^{4}$ The participants had a mean age of 40.64 years $(\mathrm{SD}=12.77), 52.28 \%$ were male, $63.68 \%$ were married or cohabiting, and most were highly educated $(59.94 \%$ had bachelor's degree or higher). Sociodemographic details are shown in Table 1.

Regarding initial contact with ayahuasca, $82.9 \%$ of the participants reported having used it for the first time through UDV, $11.56 \%$ through another religious institution, and the remaining through third parties $(4.89 \%)$, their own initiative $(0.49 \%)$, or other means $(0.16 \%)$. Of the initial motivations for having tried ayahuasca, the most frequent were spiritual $(69.54 \%)$, curiosity $(28.01 \%)$, and therapeutic $(12.21 \%)$. Family-related $(7 \%)$, recreational $(3.09 \%)$, and other motivations $(2.77 \%)$ were also mentioned.

The following usage durations were reported by the respondents: $57 \% \geqslant 10$ years, $20.52 \%$ between 5 and 10 years, $19.06 \%$ between 1 and 5 years, and 3.42\% $<1$ year. They reported using ayahuasca an average of 15.94 times in the last 6 months $(S D=7.69)$, which aligns

Table 1 Basic sociodemographic profile of ayahuasca users at a religious institution in southern Brazil $(n=614)$

\begin{tabular}{lc}
\hline Variable & $\mathrm{n}(\%)$ \\
\hline Age (years) & \\
$18-25$ & $80(13.03)$ \\
$26-35$ & $154(25.08)$ \\
$36-45$ & $170(27.69)$ \\
$46-55$ & $119(19.38)$ \\
$>55$ & $91(14.82)$ \\
Total & $614(100.00)$ \\
Mean (SD) & $40.64(12.77)$ \\
Minimum-maximum & $18-75$ \\
& \\
Sex & \\
Male & $321(52.28)$ \\
Female & $293(47.72)$ \\
Total & $614(100.00)$ \\
Marital status & \\
Single & $163(26.55)$ \\
Married & $376(61.24)$ \\
Divorced/separated & $48(7.82)$ \\
Cohabiting & $15(2.44)$ \\
Other & $12(1.95)$ \\
Total & $614(100.00)$ \\
& \\
Degree of education & \\
< Bachelor's & $245(39.97)$ \\
$>$ Bachelor's & $368(60.03)$ \\
Total & $613(100.00)$ \\
\hline SD = standard deviation. &
\end{tabular}


Table 2 Frequency and persistence of adverse physical and psychological effects experienced by participants when using ayahuasca $(n=614)$

\begin{tabular}{|c|c|c|c|c|c|c|}
\hline \multirow{2}{*}{ Adverse effects } & \multicolumn{5}{|c|}{ Frequency of occurrence } & \multirow{2}{*}{ Persistence* } \\
\hline & Never & Rarely & Occasionally & Often & Always & \\
\hline \multicolumn{7}{|l|}{ Physical } \\
\hline Diaphoresis & 354 (57.65) & $193(31.43)$ & $59(9.61)$ & $7(1.14)$ & $1(0.16)$ & $8(1.30)$ \\
\hline Diarrhea & $95(15.47)$ & 199 (32.41) & $274(44.63)$ & $38(6.19)$ & $8(1.30)$ & $101(16.45)$ \\
\hline Dizziness & $236(38.44)$ & $214(34.85)$ & $143(23.29)$ & $18(2.93)$ & $3(0.49)$ & $40(6.51)$ \\
\hline Dry mouth & $315(51.30)$ & $154(25.08)$ & 115 (18.73) & $22(3.58)$ & $8(1.30)$ & $30(4.89)$ \\
\hline Headache & $350(57.00)$ & 192 (31.27) & $69(11.24)$ & $3(0.49)$ & $0(0.00)$ & $46(7.49)$ \\
\hline Nausea & $52(8.47)$ & 127 (20.68) & 360 (58.63) & $68(11.07)$ & $7(1.14)$ & $129(21.01)$ \\
\hline Shivers & $195(31.76)$ & $148(24.10)$ & $222(36.16)$ & $39(6.35)$ & $10(1.63)$ & $9(1.47)$ \\
\hline Tachycardia & $182(29.64)$ & $175(28.50)$ & 200 (32.57) & 47 (7.65) & 10 (1.63) & $9(1.47)$ \\
\hline Tinnitus & 166 (27.04) & 165 (26.87) & 231 (37.62) & $41(6.68)$ & 11 (1.79) & $23(3.75)$ \\
\hline Tremor & 177 (28.83) & 191 (31.11) & 209 (34.04) & 31 (5.05) & $6(0.98)$ & $12(1.95)$ \\
\hline Vomiting & $20(3.26)$ & $94(15.31)$ & 421 (68.57) & 72 (11.73) & $7(1.14)$ & 75 (12.21) \\
\hline \multicolumn{7}{|l|}{ Psychological } \\
\hline Anxiety & 361 (58.79) & 162 (26.38) & $86(14.01)$ & $3(0.49)$ & $2(0.33)$ & $14(2.28)$ \\
\hline Disorientation & 459 (74.76) & 120 (19.54) & $34(5.54)$ & $0(0.0)$ & $1(0.16)$ & 93 (15.15) \\
\hline Distress & 380 (61.89) & $156(25.41)$ & 74 (12.05) & $3(0.49)$ & $1(0.16)$ & $19(3.09)$ \\
\hline Drowsiness & 162 (26.38) & 166 (27.04) & $253(41.21)$ & $25(4.07)$ & $8(1.30)$ & $17(2.77)$ \\
\hline Irritability & 498 (81.11) & $93(15.15)^{\prime}$ & $23(3.75)$ & $0(0.00)^{\prime}$ & $0(0.00)$ & $4(0.65)^{\prime}$ \\
\hline Restlessness & 362 (58.96) & $158(25.73)$ & 83 (13.52) & $10(1.63)$ & $1(0.16)$ & $23(3.75)$ \\
\hline
\end{tabular}

Data presented as $\mathrm{n}(\%)$.

* Participants who reported that the adverse effects persisted after the ceremony was over. The total duration of these persistent effects was not considered.

with previous findings that UDV members attend an average of 34.99 ceremonies annually. ${ }^{4}$

Among the reported physical effects, the most common were vomiting and nausea, with $96.74 \%$ and $91.53 \%$ of participants having experienced these effects at least once, respectively. These were also the most frequently experienced effects, with $12.87 \%$ reporting vomiting and $12.21 \%$ reporting nausea almost every time or every time they used ayahuasca. Diarrhea, shivers, tachycardia, tremor, and tinnitus were other frequent effects, with $5-10 \%$ of the participants experiencing them almost every time or every time they used ayahuasca. Persistent physical effects were reported by $41.86 \%$ of the participants, of whom the daily lives of $3.89 \%$ (10 individuals) were reasonably $(n=9)$ or very $(n=1)$ affected in a negative way. This study did not try to determine in what specific way the persistent effects negatively affected users. "Persistent adverse effects" means they continued after the ceremony was over, although the exact duration was not specified and could vary according to the subjective interpretation of each participant. The prevalence of persistent adverse effects is presented in Table 2.

Adverse psychological effects seem to be less common, and the only one experienced by more than $5 \%$ of the participants almost every time or every time they use ayahuasca was drowsiness (5.37\%). However, persistent adverse psychological effects were reported by $20.68 \%$ of the participants (127 individuals), of which $11.81 \%(n=15)$ indicated that their daily lives were moderately $(n=14)$ or very $(n=1)$ negatively affected by these persistent effects. No statistically significant difference in the persistence of physical or psychological effects was found between new and long-term users.

Regarding the severity of adverse effects, five individuals, or $0.81 \%$ of the total sample, required some form of medical assistance due to a persistent adverse physical effect, and four individuals, or $0.65 \%$ of the total sample, required assistance for a persistent adverse psychological effect. Persistent adverse effects that led participants to seek medical assistance included: irritability (two participants), anxiety (one participant), tinnitus (one participant), and nausea (one participant). Some participants had more than one persistent effect: one had vomiting and headaches, one had drowsiness and distress, one had nausea, headaches and dizziness, and one had nausea, vomiting, and diarrhea.

Five of these participants provided details about the circumstances that led them to seek medical assistance. Two interpreted the adverse effects as exacerbated symptoms of preexisting conditions (panic syndrome and anxiety disorder, the latter aggravated by grief and other personal stressors). Despite the adverse effects, both of these individuals considered ayahuasca helpful in the healing process. One participant had persistent nausea, which had no negative impact on daily life and improved with a treatment prescribed to facilitate digestion. The participant with persistent tinnitus reported having a magnesium deficiency, which was treated. One participant with persistent drowsiness and distress was diagnosed with bipolar disorder, but perceived symptoms of the condition prior to using ayahuasca for the first time. After treatment and a period of ayahuasca cessation, this participant reported being stable and was using ayahuasca again without further problems. There were no emergency cases, and all five participants recovered from the persistent effects. Four of them continued to use ayahuasca.

These findings suggest that despite occurring frequently, the adverse effects were mostly transient, self-limited, and of low severity, presenting little overall risk. The frequency of all adverse effects is presented in Table 2. 
A subgroup of 50 participants $(8.14 \%$ of the sample) reported being diagnosed with a psychiatric disorder. This is below the prevalence of common mental disorders in Brazil, which remains above $20 \%$ in different studies. ${ }^{20,21}$ The most prevalent disorders reported by participants were depressive disorder $(n=25)$, anxiety disorder $(n=19)$, bipolar disorder $(n=11)$, substance use disorder $(n=9)$, and attention deficit hyperactivity disorder $(n=5)$. Eating disorder $(n=4)$, obsessive-compulsive disorder $(n=2)$, personality disorder $(n=2)$, and schizophrenia $(n=1)$ were also reported. Depression and anxiety, the most common mental disorders worldwide, were reported by 4.07 and $3.09 \%$ of all participants, respectively. This is lower than the Brazilian average, which is $5.8 \%$ and $9.3 \%$ of the population for depression and anxiety, respectively. ${ }^{22}$ This relatively low prevalence of mental disorders among the respondents could be attributed, at least in part, to the religious practices inherent to the context, since spirituality can be a strong mental health protective factor. ${ }^{1,23}$

Psychiatric medication use was reported by $48 \%$ of the participants in this subgroup. Many participants reported more than one psychiatric diagnosis: 10 participants reported two different diagnoses, one participant reported three, two participants reported four, one participant reported five, and two participants reported six different psychiatric diagnoses. An attempt to probe the severity of these disorders revealed that $22 \%$ of the respondents with self-reported psychiatric diagnoses required psychiatric hospitalization at some point to treat their condition. This information can only serve as an indicator of the impact the disturbance had on the life of each affected participant; follow-up investigation is needed to clarify the circumstances of hospitalization.

Participants with a reported psychiatric diagnosis experienced adverse effects more frequently than those without one. Pearson's chi-square test was used to compare how often participants of both groups experienced adverse effects when using ayahuasca. Significant differences were found between participants with and without a psychiatric diagnosis for tachycardia (at least occasionally: $62 \%$ vs. $40.07 \%$ [ $p=0.003]$ ), tremors (often or every time: $16 \%$ vs. $5.14 \%[p=0.007])$, dizziness (often or every time: $12 \%$ vs. $2.66 \%$ [p $<0.001]$ ), and drowsiness (often or every time: $8 \%$ vs. $5.14 \%$ [p = $0.007])$. It is not clear to what extent the reported adverse effects were caused or intensified by ayahuasca, since many of these effects could have been symptoms of underlying psychiatric conditions. Further research is required to determine how ayahuasca might influence each psychiatric disorder.

The persistence of adverse effects after using ayahuasca was analyzed using Fisher's exact test. At least one adverse physical effect persisted in $56 \%$ of the participants with a psychiatric diagnosis, compared to $44 \%$ of those without one $(p=0.025)$. At least one adverse psychological effect persisted in $40 \%$ of the participants with a psychiatric diagnosis, compared to $18.97 \%$ of those without one $(p=0.012)$. These results do not differentiate whether the adverse effects were caused by ayahuasca, the underlying psychiatric conditions, or an interaction of both.
Thirty-one participants reported using some psychiatric medication, of whom $87.1 \%$ reported being informed about possible interactions with ayahuasca. Twenty-five participants $(4.07 \%$ of the sample) reported that they were taking antidepressants, which is lower than the $6.87^{24}$ and $9.3 \%{ }^{25}$ prevalence found in other population studies in Brazil. This is an approximate comparison, since no studies with similar samples could be found. Pearson's chi-square test was performed to evaluate the influence of psychiatric drugs on the frequency of reported adverse effects. The hypothesis was that concomitant ayahuasca use could cause changes in monoamine degradation, especially serotonin, to the point of manifesting more intense or frequent adverse effects. To limit the scope of drug action, only antidepressants were considered for this analysis. Nine participants were using more than one antidepressant at the time. The antidepressants used by the participants were bupropion, citalopram, desvenlafaxine, escitalopram, fluoxetine, mirtazapine, sertraline, trazodone, and venlafaxine, some of which have already been implicated in cases of serotonin toxicity. ${ }^{26}$ Two participants did not specify which antidepressants they used. All these were considered as a single group to perform a preliminary analysis.

No statistically significant difference $(p>0.05)$ in adverse effects was found between participants who used antidepressants and those who did not. This suggests that there is no relationship between the variables. Larger samples are required to confirm this finding, since the low number of antidepressant users prevents analysis of individual drugs. There was a statistical trend toward the persistence of adverse psychological effects according to Fisher's exact test ( $p=0.055)$ : $36 \%$ of participants who used antidepressants reported persistent effects, compared to $20.17 \%$ of those who did not. Users may have overlapping psychiatric symptoms and adverse effects caused by prescription drugs and/or ayahuasca. The subjective nature of these symptoms prevents the identification of a single causative agent, which limits the analysis.

Concerning the concomitant use of prescription drugs (psychiatric or otherwise), the participants were asked about modifications or interruptions in their medication use. A total of $43(7 \%)$ participants reported having temporarily modified their use of a prescription drug to use ayahuasca, and $10(1.63 \%)$ reported having done so on a permanent basis. These modifications represent changes in dosage, interval, or number of doses. Eighteen (2.93\%) participants temporarily interrupted their use of a prescription drug to take ayahuasca, and another 10 (1.63\%) reported having done so on a permanent basis. The motivation for these modifications and interruptions was beyond the scope of this study; it is possible the participants intended to avoid presumed drug interactions or were attempting to replace conventional therapies with ayahuasca use. This could be the case in disorders such as depression, drug abuse, and anxiety, for which the therapeutic use of ayahuasca is being studied. ${ }^{27-34}$

Regarding safety measures, the participants were questioned about the orientation and health evaluation they received prior to using ayahuasca for the first time, as well as any assistance they received during the event. 
Of the participants with valid answers for this item $(n=508)$, $61.61 \%$ received a physical and mental health evaluation prior to their first use (the nature of this evaluation was not specified). A total of $74.81 \%$ of these participants evaluated the orientation as satisfactory or very satisfactory $(n=540)$, and $88.95 \%$ evaluated the assistance received as satisfactory or very satisfactory $(n=552)$.

Since UDV has played a pivotal role in Brazilian and international ayahuasca regulation, ensuring safe practice appears to be a permanent concern, especially with the growing public interest. ${ }^{35,36}$ For this reason, the implementation of harm reduction measures over time was explored. Fisher's exact test was used to compare outcomes for "orientation, assistance, and physical and mental health evaluation" received upon first ayahuasca use in relation to "time since first use." This procedure tested the hypothesis that harm reduction measures were more prevalent among participants who began ayahuasca use more recently than among long-term users. A significant increase in physical and mental health evaluations was found ( $p<0.001)$. Among the participants who provided valid answers $(n=508), 76.92 \%$ of those who started using ayahuasca $\leqslant 5$ years ago $(n=117)$ underwent a physical and mental health evaluation prior to first use, compared to $57.03 \%$ of those whose first use was $>5$ years ago $(n=391)$.

Among those who provided valid answers $(n=540)$, there was a statistically significant increase $(p=0.005)$ over time in the percentage of participants who received an orientation prior to first ayahuasca use: $96.88 \%$ vs. $89.56 \%$ for those whose first use was $\leqslant 5$ years ago $(n=128)$ and $>5$ years ago $(n=412)$, respectively. No significant difference was found in assistance received during first use between these two groups. In this analysis, only basic quantitative aspects were considered; different contexts of initial contact with ayahuasca were ignored.

\section{Discussion}

In general, risk reduction measures for ayahuasca use were widespread and positively evaluated in the context of UDV. Screening prospective users is necessary to identify risk factors, allowing susceptible individuals to be more closely monitored or advised against use. The monitoring of individuals under the effect of ayahuasca, especially inexperienced users, is essential to detect and adequately intervene in the emergence of unfavorable events, whether due to drug interactions, co-occurring psychiatric conditions, or an unanticipated event.

In the analyzed context, concomitant use of ayahuasca and antidepressants does not appear to be associated with an increased occurrence of adverse effects, although it may influence the persistence of adverse psychological effects. These results support the hypothesis that the MAOIs in ayahuasca have a mild, short-lived effect and are unlikely to cause severe interactions when used responsibly. Given that drug interactions and adverse reactions often depend on individual characteristics, the task of establishing safety when combining ayahuasca with specific medications remains a challenge. The modification or interruption of prescribed drugs should be better investigated in future studies, since the lack of details about motivations and professional supervision prevents us from drawing conclusions.

Our findings should be considered with caution, since no conventional $\mathrm{MAOI}$ or tricyclic antidepressant users were identified, our results do not apply to these drug classes. This is also the case for other substances that were not considered in this study. Regardless of their legal status, recreational drugs with inherent associated risks, such as alcohol, cannabis, MDMA, lysergic acid diethylamide, etc. should be avoided by ayahuasca users until their co-use with ayahuasca has been demonstrated to be safe.

Individuals with a psychiatric diagnosis are advised to inform the healthcare professional responsible for their treatment about the decision to use ayahuasca. In the absence of contraindication, these users should be supervised and periodically reassessed. Each condition may be influenced differently by ayahuasca: while some may be negatively affected, others may benefit from its therapeutic potential. Ongoing investigations are gathering evidence about the therapeutic use of ayahuasca and its components for treating depression, ${ }^{27-31}$ anxiety, ${ }^{29-31}$ and substance abuse disorder, ${ }^{31-34}$ among other conditions. Despite the lack of guidelines or approved treatments, ayahuasca is already attracting interest from individuals eager to benefit from its therapeutic potential, as can be attested by the fact that $12.21 \%$ of the respondents reported that one of their initial motivations for using ayahuasca was therapeutic.

As study limitations, many variables were not exhaustively explored, which is to be expected in a broadly aimed project on a complex subject. For example, hospitalization history can only serve as an indicator of psychiatric disorder severity; validated metrics should be employed in future studies, and there should be an attempt to determine whether the hospitalizations were in any way related to ayahuasca use. The same precautions should be taken regarding persistent adverse effects and the need for medical attention, a topic that requires further investigation. It must be pointed out that the validity of any results is subject to the particularities of the sample, considering the specific population studied and its context. Comparing the prevalence of risk factors in this sample to that of the general population is limited by a lack of epidemiological data obtained using standardized instruments and eligibility criteria. The over-representation of long-term users, for example, hinders generalization of the results to different groups, especially new users. Adverse effects in ayahuasca-naïve subjects may vary considerably from those of the individuals evaluated in this sample. It is worth mentioning that nausea and vomiting, despite being considered adverse effects in medical terms, may assume an important ritualistic role among indigenous and religious ayahuasca cultures, in which they are sometimes not seen as adverse, but beneficial and even desirable. ${ }^{37}$

Using an online questionnaire limits participation by individuals with low literacy or those who have no access to or little familiarity with the technological means necessary to participate. Likewise, some individuals may have 
abstained from participating due to mistrust or worries about negative repercussions, although such could hardly be the case since the survey was disseminated through UDV and had guaranteed anonymity, even from the institution itself. Moreover, it cannot be guaranteed that the entire population was exposed to information about the survey, since this would require unauthorized identification. Despite these limitations, however, a sample of almost $40 \%$ of the known population was gathered with minimal resources.

Finally, collaborating with UDV was essential to reach ayahuasca users, although this strategy has the unavoidable bias of selecting participants from a population already prone to benefit from ayahuasca use. Therefore, the collected data can only provide limited evidence about the safety of ayahuasca for the general population. However, since the study is expanding existing knowledge, it serves to build understanding about the issue. Future studies can use our results to target more specific problems. The development of a standardized, validated tool for acquiring the necessary information to establish the safety of ayahuasca use in any given context would be of immense value.

In conclusion, many factors are implicated in the safety of ayahuasca use. The existence of widely employed and well-evaluated safety measures is indicative of responsible practice by UDV. These measures are of special importance in situations where the risk of drug interactions and negative influence on co-occurring psychiatric disorders is still unknown. Hence, they should always be encouraged and improved.

The adverse effects of ayahuasca, despite being frequent, are mostly mild and seem to be well tolerated by users. Many participants reported the persistence of physical $(41.86 \%)$ or psychological $(20.68 \%)$ adverse effects following the ayahuasca ceremony, and the daily lives of a minority were negatively affected in some way. Furthermore, $1.47 \%$ of the 614 participants required medical assistance due to a persistent adverse effect. Nevertheless, these adverse effects seem to be predominantly self-limited and do not appear to pose a serious risk to healthy users.

Given that the safety of ayahuasca use among individuals with psychiatric disorders or who use psychiatric medications has not been well established and that our findings indicate the occurrence of adverse effects, it is crucial to develop appropriate tools for screening and evaluating such cases. While antidepressant use had no significant influence on the frequency of adverse effects, psychiatric medication users should remain wary of possible drug interactions and inform the professional responsible for their treatment about their intention to use ayahuasca.

\section{Acknowledgements}

RGS is fellow of the Programa Nacional de Pós-Doutorado (Coordenação de Aperfeiçoamento de Pessoal de Nível Superior, Brazil). JCB is the scientific director of the International Center for Ethnobotanical Education, Research and Services, a non-profit organization that promotes the scientific research of plant hallucinogens such as ayahuasca and ibogaine. JEH receives a Conselho Nacional de Desenvolvimento Científico e Tecnológico (Brazil) productivity fellowship award.

The authors would like to thank professors Athany Gutierres and Bruna Chaves Lopes from Universidade Federal da Fronteira Sul for proofreading, guidance, and enlightening discussions; we thank Ariane Rauber and Jessica Longo from Universidade de Passo Fundo, and Marina Monteiro from Universidade Federal da Fronteira Sul, for their diligence in helping whenever needed. Our deep gratitude to the members of União do Vegetal, who kindly answered the questionnaire, and especially to Cesar Volpato and the members of UDV's medical and scientific department, whose help was fundamental. Recognition is due to all those who produce, maintain, and distribute free open source software, as well as to those who remove barriers in the way of science.

\section{Disclosure}

The authors report no conflicts of interest.

\section{References}

1 Grob CA, Mckenna DJ, Callaway JC, Brito GS, Neves ES, Oberlaender $G$, et al. Human psychopharmacology of hoasca, a plant hallucinogen used in ritual context in Brazil. J Nerv Ment Dis. 1996; 184:86-94.

2 Bernardino-Costa J, Da Silva FM. Construindo o mundo da hoasca: a organização da união do vegetal. In: Hoasca: ciência, sociedade e meio ambiente. Campinas: Mercado das Letras; 2011. p. 21-42.

3 Centro Espírita Beneficente União do Vegetal in the United States. 2019 Nov 19 [cited 2019 Nov 19]. udvusa.org

4 Barbosa PC, Tófoli LF, Bogenschutz MP, Hoy R, Berro LF, Marinho $E A$, et al. Assessment of alcohol and tobacco use disorders among religious users of ayahuasca. Front Psychiatry. 2018;9:136.

5 Soares DB, Duarte LP, Cavalcanti AD, Silva FC, Braga SD, Lopes MT, et al. Psychotria viridis: chemical constituents from leaves and biological properties. An Acad Bras Cienc. 2017;89:927-38.

6 Carbonaro TM, Gatch MB. Neuropharmacology of N,N-dimethyltryptamine. Brain Res Bull. 2016;126:74-88.

7 Strassman RJ, Qualls CR, Berg LM. Differential tolerance to biological and subjective effects of four closely spaced doses of N,Ndimethyltryptamine in humans. Biol Psychiatry. 1996;39:784-95.

8 Tipton KF, Boyce S, O'Sullivan J, Davey GP, Healy J. Monoamine oxidases: certainties and uncertainties. Curr Med Chem. 2004; 11:1965-82.

9 Domínguez-Clavé E, Soler J, Elices M, Pascual JC, Álvarez E, Revenga MF, et al. Ayahuasca: pharmacology, neuroscience and therapeutic potential. Brain Res Bull. 2016;126:89-101.

10 Vetulani J, Nalepa I. Antidepressants: past, present and future. Eur J Pharmacol. 2000;405:351-63.

11 Yamada M, Yasuhara $\mathrm{H}$. Clinical pharmacology of MAO inhibitors: safety and future. Neurotoxicology. 2004;25:215-21.

12 McCabe-Sellers BJ, Staggs CG, Bogle ML. Tyramine in foods and monoamine oxidase inhibitor drugs: a crossroad where medicine, nutrition, pharmacy, and food industry converge. J Food Compost Anal. 2006;19(Suppl): S58-65.

13 Volpi-Abadie J, Kaye AM, Kaye AD. Serotonin syndrome. Ochsner J. 2013;13:533-40.

14 Callaway JC, Grob CS. Ayahuasca preparations and serotonin reuptake inhibitors: a potential combination for severe adverse interactions. J Psychoactive Drugs. 1998;30:367-9.

15 Gable RS. Risk assessment of ritual use of oral dimethyltryptamine (DMT) and harmala alkaloids. Addiction. 2007;102:24-34.

16 Riba J, Rodríguez-Fornells A, Urbano G, Morte A, Antonijoan R, Montero $M$, et al. Subjective effects and tolerability of the South 
American psychoactive beverage ayahuasca in healthy volunteers. Psychopharmacology (Berl). 2001;154:85-95.

17 Callaway JC, McKenna DJ, Grob CS, Brito GS, Raymon LP, Poland $\mathrm{RE}$, et al. Pharmacokinetics of hoasca alkaloids in healthy humans. J Ethnopharmacol. 1999;65:243-56.

18 dos Santos RG. Safety and side effects of ayahuasca in humans-an overview focusing on developmental toxicology. J Psychoactive Drugs. 2013;45:68-78.

19 Werneke U, Jamshidi F, Taylor DM, Ott M. Conundrums in neurology: diagnosing serotonin syndrome - a meta-analysis of cases. BMC Neurol. 2016;16:97.

20 dos Santos EG, de Siqueira MM. Prevalência dos transtornos mentais na população adulta brasileira: uma revisão sistemática de 1997 a 2009. J Bras Psiquiatr. 2010;59:238-46.

21 Nunes MA, Pinheiro AP, Bessel M, Brunoni AR, Kemp AH, Benseñor $\mathrm{IM}$, et al. Common mental disorders and sociodemographic characteristics: baseline findings of the Brazilian longitudinal study of adult health (ELSA-Brasil). Braz J Psychiatry. 2016;38:91-7.

22 World Health Organization (WHO). Depression and other common mental disorders: global health estimates. 2017 [cited 2020 Jun 23]. apps.who.int/iris/handle/10665/254610

23 Moreira-Almeida A, Koenig HG, Lucchetti G. Clinical implications of spirituality to mental health: review of evidence and practical guidelines. Braz J Psychiatry. 2014;36:176-82.

24 Brunoni AR, Nunes MA, Figueiredo R, Barreto SM, da Fonseca MJ, Lotufo PA, et al. Patterns of benzodiazepine and antidepressant use among middle-aged adults. The Brazilian longitudinal study of adult health (ELSA-Brasil). J Affect Disord. 2013;151:71-7.

25 Garcias CM, Pinheiro RT, Garcias GL, Horta BL, Brum CB. [Prevalence of antidepressant use and associated factors among adults in Pelotas, Rio Grande do Sul State, Brazil, 2006]. Cad Saude Publica. 2008;24:1565-71.

26 Moss MJ, Hendrickson RG; Toxicology Investigators Consortium (ToxIC). Serotonin toxicity: associated agents and clinical characteristics. J Clin Psychopharmacol. 2019;39:628-33.
27 Osório FL, Sanches RF, Macedo LR, dos Santos RG, Maia-de-Oliveira JP, Wichert-Ana L, et al. Antidepressant effects of a single dose of ayahuasca in patients with recurrent depression: a preliminary report. Braz J Psychiatry. 2015;37:13-20.

28 da Silva FS, Silva EA, de Sousa GM Jr, Maia-de-Oliveira JP, SoaresRachetti VP, de Araujo DB, et al. Acute effects of ayahuasca in a juvenile non-human primate model of depression. Braz J Psychiatry. 2019;41:280-8.

29 Dos Santos RG, Osório FL, Crippa JA, Hallak JE. Antidepressive and anxiolytic effects of ayahuasca: a systematic literature review of animal and human studies. Braz J Psychiatry. 2016;38:65-72.

30 Muttoni S, Ardissino M, John C. Classical psychedelics for the treatment of depression and anxiety: a systematic review. J Affect Disord. 2019;258:11-24.

31 Dos Santos RG, Bouso JC, Alcázar-Córcoles MÁ, Hallak JE. Efficacy, tolerability, and safety of serotonergic psychedelics for the management of mood, anxiety, and substance-use disorders: a systematic review of systematic reviews. Expert Rev Clin Pharmacol. 2018;11:889-902.

32 Winkelman M. Psychedelics as medicines for substance abuse rehabilitation: evaluating treatments with LSD, peyote, ibogaine and ayahuasca. Curr Drug Abuse Rev. 2014;7:101-16.

33 Tófoli LF, de Araujo DB. Treating addiction: perspectives from EEG and imaging studies on psychedelics. Int Rev Neurobiol. 2016;129:157-85.

34 Sessa B, Johnson MW. Can psychedelic compounds play a part in drug dependence therapy?. Br J Psychiatry. 2015;206:1-3.

35 Labate BC, Feeney K. Ayahuasca and the process of regulation in Brazil and internationally: implications and challenges. Int J Drug Policy. 2012;23:154-61.

36 Heise CW, Brooks DE. Ayahuasca exposure: descriptive analysis of calls to US poison control centers from 2005 to 2015. J Med Toxicol. 2017;13:245-8.

37 Labate B, de Rose I, dos Santos R. Ayahuasca religions: a comprehensive bibliography and critical essays. Santa Cruz: Multidisciplinary Association for Psychedelic Studies; 2009. 